Proceedings of the 2012 Winter Simulation Conference

C. Laroque, J. Himmelspach, R. Pasupathy, O. Rose, and A.M. Uhrmacher, eds

\title{
METHODOLOGY TO BEST EXTEND AMHS FOR SITE EXPANSION
}

\author{
Gabriel Gaxiola \\ Eric Christensen \\ Christian Hammel \\ Manufacturing Technology \\ GLOBAFOUNDRIES U.S. Inc., \\ 400 Stone Break Rd, Malta, NY \\ Institute of Material Handling and \\ Industrial Engineering \\ Technische Universität Dresden \\ D-01062 Dresden, GERMANY
}

\author{
Paul Stachura \\ Stachura Enterprises, LLC \\ Austin, TX
}

\begin{abstract}
As companies grow their capacity in multiple buildings there are increasing challenges with the automated material handling systems (AMHS) used to transport the wafers between two or more facilities. In some cases, the links used to perform these transports can become a constraint for the entire system. The problem grows more difficult as the expansion plan extends further into the future, making it harder to predict throughput requirements. This study discusses a particular throughput prediction tool as well as different approaches for evaluating designs. The approaches discussed include: integrated vehicle/conveyor model using static tool (Network approach), segregated dynamic models for conveyor/vehicle system, and integrated vehicle/conveyor dynamic model. The pros/cons of these approaches are discussed based on different use cases. The paper finishes by discussing the strategic advantages of factories performing expansion analysis early in the design of the factory and the importance to continue validating and improving these methods.
\end{abstract}

\section{INTRODUCTION}

The first fab on a semiconductor manufacturing site is often not the last fab for the site. As demand for the company increases, it is often advantageous to leverage the existing infrastructure for the additional capacity a new fab can provide. Just as the water mains, electrical power, and transportation needs encompass the long term capacity needs when a site plan is developed, the AMHS design should take into consideration potential expansion requirements for the site. Although it is possible to design new fabs to operate completely independent from the original fab, there are often cost advantages to adding additional factories by leveraging capacity in the already existing fab(s). This approach can create a flow of material back and forth between new and old fab(s) during the ramp up period through full production. To support this flow of material, AMHS designers face several questions that need to be addressed:

- How big should interconnects be?

- How many and where should they be located?

- What are the dimensions of the hallways or bridges that would support them?

Also, the designers will need to ensure the delivery systems will meet required inter-fab throughput without disrupting the flow inside the original fab or the new fab. If these questions are not addressed success- 
fully early in the design process, the site could incur additional AMHS cost throughout its life and could negatively impact the combined potential capacity of the entire campus.

Designers are tasked to address these issues in the most efficient way based on time constraints and resources available. This paper discusses this effort in regards to input definition and design evaluation/output generation. There are several approaches that vary in terms of complexity and usefulness depending on the use case. This paper's intent is to shed some light regarding which approach to use for which cases.

The remainder of the paper is outlined as follows: Section II will summarize related work, Section III will describe an interfab moves prediction model, Section IV will discuss three different design evaluation methodologies including pros and cons, and Section V will present the summary and conclusions.

\section{RELATED WORK}

Literature research shows a significant number of publications in the area of AMHS modelling for design, forecast and decision making in a single fab or sub-sections of a single fab. Concerning vehicle-based models, Jimenez et al. (2002) looks at an AMHS design based on rails and lifters in different floors and tries to minimize average lot-delivery time. Sturm et al. (2003) outlines the planning approach for two AMHS designs used for interbay transportation in $200 \mathrm{~mm}$ wafer fabs with regard to good performance and robustness of the system. Nazzal \& McGinnis (2006) proposes an analytical model using an extended Markov chain useful in the design of AMHS. Gaxiola et al. (2004) evaluates the benefit of using a redundant overhead shuttle (OHS) system in combination with an interconnected overhead transport (OHT) system to support automated movement of non-production wafers. With regards to conveyor-based models, Miller et al. (2011) discusses the design and test of conveyor-based AMHS configurations with emphasis on comparing centralized versus distributed storage systems. Also, Nazzal et al. (2008) proposes an analytical model for the design of a conveyor-based AMHS to estimate the work-in-process on the conveyor and assess the system stability.

There is an additional set of publications that focuses on linked or semi-linked AMHS/factory models. Jimenez et al. (2010) study AMHS productivity detractors affecting small lot manufacturing (SLM) and use linked simulation models for testing the 12 and 25 wafer-lot scenarios. Kiba et al. (2009) presents a detailed simulation model of the production system and AMHS for a $300 \mathrm{~mm}$ semiconductor plant and discusses some of the studies performed with this model, Jimenez et al (2005) discusses capacity models with less detailed AMHS representations which can generate accurate system predictions in comparison to the values produced by fully integrated models while Jimenez et al (2008) identifies a method for classifying a fab model by the level of capacity detail, the level of AMHS detail, or the level of capacity/AMHS detail.

Kohn et al. (2009) considers future performance prediction using simulation techniques different to discrete event simulation and several optimization approaches and Kondo (2008) discusses how AMHS solutions for SLM should stress more transport capacity and shorter Carrier Exchange Time (CET) rather than shorter delivery time. Zimmerhackl et al. (2007) analyzes the effects of SLM on equipment operation and fab performance and describe options for carrier handling and AMHS operation to maximize the productivity benefits of SLM.

In general, none of these publications deal with the design of AMHS extensions into multiple buildings and therefore there is an interest in presenting the work that has been conducted in this area.

\section{INTERFAB MOVES PREDICTION MODEL}

In order to come up with the design of the facilities AMHS interlinks, the first question to be answered is: "How many moves need to be handled back and forth by the interlink(s)?" The interlink design should be able to accommodate an immediate first-step expansion as well as any possible future expansions within the fab campus. It is recognized that it is possible to accomplish these predictions using factory models that depict in detail the flows, equipment, and logic that will be used in the fabs to trigger such moves 
(based on load balancing, staging vs. travel times, randomness of down times, etc), but creating such detailed models takes time and requires initial assumptions that will be continually changing during the entire design lifecycle. Instead, a simpler probabilistic model is considered to obtain these interfab move estimates.

A probabilistic spread sheet model to calculate the quantity of movements between buildings can be developed based on three inputs: a process flow, the quantity of required equipment in each building, and the start rate for the site. A single process flow may be used to represent all flows if its share of the total throughput is large enough. Equipment processing times and capacities may be assumed to be sufficient for the start rate and all tools to have the same probability of being used (depending on a certain granularity of tool families). Based on this, the material distribution can be analysed step by step through the entire process flow as shown in table 1. In each step, material is distributed to the buildings according to the required equipment location and quantity. If there are 32 of 65 tools in building 1 able to do the first process step in table 1, nearly half of the material is assumed to be processed in this building. For choosing the equipment in the next step, priority is given to tools within the same building. Anything that can be processed without a building change, remains with the equipment in that building. The remaining material needed to be processed (based on the respective sampling rate) is being evenly distributed to the equipment in other buildings. This causes movements between buildings which are tabulated in a from/to matrix based on the share of total material that is moved (columns "In / Out moves to next step"). According to the example in Table 1, everything has to be moved out of building 3 after the first implant step and into the other building based on their respective number of tools for the second implant step. It is assumed that anything going out of one building has to go into another, therefore only the out-moves are highlighted by yellow and red colours. With this visual feedback, this probabilistic model quickly highlights the problems: equipment placement balance between buildings, process flow inefficiencies, and inter building traffic. Summarizing all inter building moves using this tool provides requirements that can be used for the interlink design. Furthermore, it can be used to automatically analyse changes in tool distribution over the buildings.

Table 1: Interfab Moves Probabilistic Prediction Model

\begin{tabular}{|c|c|c|c|c|c|c|c|c|c|c|c|c|c|c|c|}
\hline \multirow[b]{2}{*}{ Tool current Setup } & \multirow{2}{*}{$\begin{array}{l}\text { Sampling } \\
\text { rate }\end{array}$} & \multicolumn{5}{|c|}{ Tool count } & \multicolumn{4}{|c|}{ In / Out moves to next step } & \multicolumn{4}{|c|}{ Moves in module } & \multirow{2}{*}{$\begin{array}{l}\text { Total } \\
\text { building } \\
\text { change }\end{array}$} \\
\hline & & Blg 1 & $\mathrm{Blg} 2$ & Blg 3 & Blg 4 & total & Blg 1 & $\mathrm{Blg} 2$ & Blg 3 & $\mathrm{Blg} 4$ & Blg 1 & $\mathrm{Blg} 2$ & $\mathrm{Blg} 3$ & $\mathrm{Blg} 4$ & \\
\hline Masking & $100 \%$ & 32 & 13 & 20 & 0 & 65 & $2 \%$ & $1 \%$ & $-3 \%$ & $0 \%$ & $16 \%$ & $6 \%$ & $7 \%$ & $0 \%$ & $3 \%$ \\
\hline Optical Review & $32 \%$ & 5 & 2 & 2 & 0 & 9 & $-5 \%$ & $1 \%$ & $4 \%$ & $0 \%$ & $8 \%$ & $5 \%$ & $7 \%$ & $0 \%$ & $5 \%$ \\
\hline After Develop Inspection & $26 \%$ & 6 & 5 & 8 & 0 & 19 & $2 \%$ & $0 \%$ & $-2 \%$ & $0 \%$ & $29 \%$ & $14 \%$ & $17 \%$ & $0 \%$ & $2 \%$ \\
\hline Overlay & $63 \%$ & 9 & 4 & 5 & 0 & 18 & $25 \%$ & $-11 \%$ & $-14 \%$ & $0 \%$ & $23 \%$ & $0 \%$ & $0 \%$ & $0 \%$ & $25 \%$ \\
\hline Critical Dimension Measurement & $48 \%$ & 6 & 0 & 0 & 0 & 6 & $-4 \%$ & $2 \%$ & $3 \%$ & $0 \%$ & $12 \%$ & $3 \%$ & $3 \%$ & $0 \%$ & $4 \%$ \\
\hline Defect Inspection & $22 \%$ & 8 & 3 & 4 & 0 & 15 & $-2 \%$ & $0 \%$ & $2 \%$ & $0 \%$ & $7 \%$ & $2 \%$ & $2 \%$ & $0 \%$ & $2 \%$ \\
\hline Defect Review & $13 \%$ & 6 & 2 & 4 & 0 & 12 & $-3 \%$ & $3 \%$ & $1 \%$ & $0 \%$ & $18 \%$ & $4 \%$ & $6 \%$ & $0 \%$ & $3 \%$ \\
\hline Optical Review & $32 \%$ & 5 & 2 & 2 & 0 & 9 & $-63 \%$ & $-17 \%$ & $79 \%$ & $0 \%$ & $0 \%$ & $0 \%$ & $21 \%$ & $0 \%$ & $79 \%$ \\
\hline Implant & $100 \%$ & 0 & 0 & 2 & 0 & 2 & $27 \%$ & $42 \%$ & $-100 \%$ & $31 \%$ & $0 \%$ & $0 \%$ & $0 \%$ & $0 \%$ & $100 \%$ \\
\hline Implant & $100 \%$ & 7 & 11 & 0 & 8 & 26 & $16 \%$ & $-4 \%$ & $12 \%$ & $-24 \%$ & $27 \%$ & $38 \%$ & $0 \%$ & $7 \%$ & $28 \%$ \\
\hline Plasma Strip & $100 \%$ & 18 & 16 & 5 & 3 & 42 & $-12 \%$ & $6 \%$ & $13 \%$ & $-7 \%$ & $31 \%$ & $38 \%$ & $12 \%$ & $0 \%$ & $19 \%$ \\
\hline Wet Strip & $100 \%$ & 5 & 7 & 4 & 0 & 16 & $-11 \%$ & $6 \%$ & $5 \%$ & $0 \%$ & $20 \%$ & $44 \%$ & $25 \%$ & $0 \%$ & $11 \%$ \\
\hline
\end{tabular}

\section{DESIGN EVALUATIONS METHEDOLOGY}

Once the requirements for moves have been set, the designer will be tasked with selecting the methodology that best fits to evaluate different designs. The methodology used should be able to test the throughput requirements and also provide estimates of delivery times between tool sets in different buildings. In turn, these delivery time estimates will also influence performance expectations for interconnected fab(s) and will help create optimized algorithms for tool selection (e.g. controller should trigger a move only if expected queue time is longer than expected delivery time). In order to evaluate these designs, three different methods are considered in the following sections, followed by a comparison of the pros and cons of each. In section 4.1, the application of the Network approach based on flow analysis and weighted paths 
is discussed. Section 4.2 has a discussion of dynamic models, with discrete sections of the system modelled separately (e.g. one model for conveyor section, different models for OHT section in building A and building B). Section 4.3 discusses fully integrated AMHS dynamic models. Finally, section 4.4 reviews the pros and cons and case application of each approach.

\subsection{Integrated Static Models (Network Approach)}

Particularly in the early planning phase, there is a certain discrepancy between the level of detail necessary for dynamic simulation models regarding control logic, vehicle parameters, and transport data with the tentativeness of available information on requirements, constraints and assumptions. Thus, the dynamic simulations might promise more accurate results than actually supported by existing data and the effort of creating a dynamic simulation might be unjustified at such an early planning stage.

A network approach based on AMHS track utilization by loaded vehicles (or conveyor throughput) is another way to establish a basis for further decisions. In this more simplistic approach, the AMHS is represented by a network graph model as proposed by Hammel et al. (2008). Each intersection and potential source or sink of transports becomes a network node; the connecting tracks are the links between them. Assuming that routing decisions of the AMHS are made by some kind of shortest path algorithm, where 'shortest' may be referring to real length or some arbitrary costs assigned to the links, track utilization can be determined using sophisticated network algorithms. The Floyd-Warshall-Algorithm (Floyd 1962, Warshall 1962) for example is designed to identify all shortest paths between any two nodes in one pass. To be more exact, it actually identifies the next node to go to while at one node and heading for a certain destination node. From this matrix, the shortest paths can be generated for all needed origin-destination relationships. By generating a list of transports for a certain time period and identifying the shortest path for each single transport, the average number of transports per hour on each link is determined. This approach neglects any empty vehicle moves. Also, similar to the dynamic simulation, it is dependent on the assumptions and simplifications made when modelling transports. If these transports are generated from certain process flows, they might not include test wafers or empty FOUPs for example. So a certain safety factor has to be applied either to the transports themselves or to the resulting track utilization. Arguing that empty vehicle moves only account for a small percentage of all moves, especially in high load scenarios, the generated track utilization can be taken as acceptably realistic. Collecting maximum numbers of transports per time period through one piece of track from existing comparable systems, the comparison to the calculated numbers gives insight if the layout is functional and where bottlenecks could occur. Furthermore, this approach allows an estimation of transport times based on transport distance and, if they have an impact, number of intersections. Even though this does not take retrieval times into account, it is an acceptable estimate at this stage.

As this method is magnitudes faster in run time than conventional dynamic simulation, and networks can be manipulated quite simply, it is suitable for layout evaluation while it is still possible to change. Different layout alternatives can fast and easily be assessed and compared. Moreover, layouts can be adjusted and tested in order to get rid of over-utilization of pieces of track. Especially for the connections to other fabs, this is an essential point. Where and how to connect track to another building can make a big difference, which is not easily seen without a track utilization chart because of the underlying complexity of the transport structure. As AMHS track mostly has only one level and cannot cross on top of each other, crossing streams are often a problem. In case of fab connections, this becomes obvious if there are two connections with one lane in each direction (Figure 1). Even though maximum throughput could be expected to be doubled compared with one connection, it might in fact not be much higher. If there are two or more typical transport streams that have to cross each other at some point, this crossing area becomes the bottleneck. 


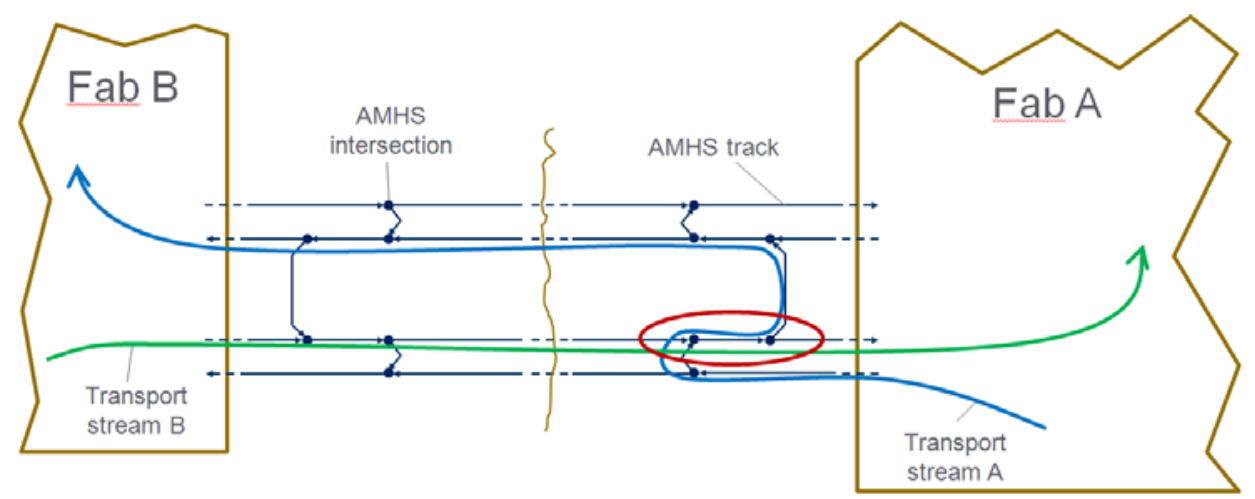

Figure 1: Crossing transport streams limiting the throughput of fab connections

Traffic has to be divided as far as possible to have only certain transport relations use each connection. Furthermore, AMHS track layout has to be adjusted to best support this traffic division. Both aspects can easily be tested by the described network approach. For traffic distribution and in addition to the mere shortest path analysis, an optimization of transport routes is also possible. Routing in state-of-the-art systems is indeed determined by 'lowest priced' paths and the underlying costs for passing pieces of track by vehicles can be adjusted. Hence, manipulation of these costs can redistribute traffic; it can be employed to lower track utilization of over-utilized tracks. Schöps \& Hammel (2012) shows that such a cost parameter optimization increased maximum throughput of the AMHS in a producing fab by up to $20 \%$ without negative impact to delivery times. Of course, there is a trade-off with delivery times in general. Distributing traffic away from shortest paths elongates the transport distance for vehicles. If this avoids passing bottlenecks, which tend to be at risk of generating traffic jams, a certain longer way should be considered. If there is no risk of traffic jams on the contrary, taking the shortest route is the method of choice. So the right balance of using shortest paths and avoiding traffic jams by decreasing track utilization has to be found. If the maximum throughput of a piece of track is known, the goal of traffic redistribution is to get all track utilizations below this limit. If this is possible, the resulting average travel distance is the benchmark of the acceptability of the considered AMHS layout.

Table 2 shows how results obtained from Network approach compare with results obtained from integrated dynamic simulation approach. The results below are presented as ratios of the results from dynamic model and network approaches for confidentiality reasons. We can see that for car-based systems, maximum throughput estimates matched very well with the results obtained with dynamic modelling, while estimates of average transport time were close. The conveyor system was modelled with Network approach as well, but with much less understanding of the operation characteristics of that system. Therefore, results obtained were considerably off when compared to results obtained from dynamic modelling.

Table 2: Network Approach vs. Integrated Dynamic Approach Results

\begin{tabular}{|l|c|c|c|}
\hline $\begin{array}{l}\text { Dynamic/Network } \\
\text { Approach Ratio }\end{array}$ & $\begin{array}{c}\text { 4 Lanes_Vehicle } \\
\text { Based }\end{array}$ & $\begin{array}{c}\text { 6 Lanes_Vehicle } \\
\text { Based }\end{array}$ & $\begin{array}{c}\text { Conveyor } \\
\text { System }\end{array}$ \\
\hline Max Throughput & 1.0 & 1.0 & 2.0 \\
\hline Avg. Transporting Time & 0.8 & 0.9 & 1.5 \\
\hline
\end{tabular}

\subsection{Segregated Dynamic Models}

When expansion planning for a given campus begins, typically the dynamic models of the initial fab are already available and therefore a decision has to be made whether to expand the initial model (as depicted in section 4.3) or create a separate model with just new fab information. Keeping the models segregated will allow for better manageability and running times, but will rely on assumptions about the other fab. 
How good those assumptions are will affect decision quality, which could add risk to this approach when compared to the fully integrated approach.

Even with this greater risk there are several aspects that make this approach attractive besides the manageability and run times mentioned before. One aspect is the data quality available for the old vs. the new fab. When expanding an existing site there is at least one fab that is already running, so all information can be gathered from real production data. The additional fab is in a rough planning phase where only assumptions on transport scenarios, control logic, and AMHS parameters can be made. Each model's results may be interpreted against the background of accuracy of the used data. The existing fab model can be extended by the transfer connections and a direct comparison to the current validated model can be drawn, so the impact of the connection to production can be directly estimated. The results of the simulation model of the new fab with transfer connections included have to be considered with a larger deviation. Figure 2 shows an example of segregated models.

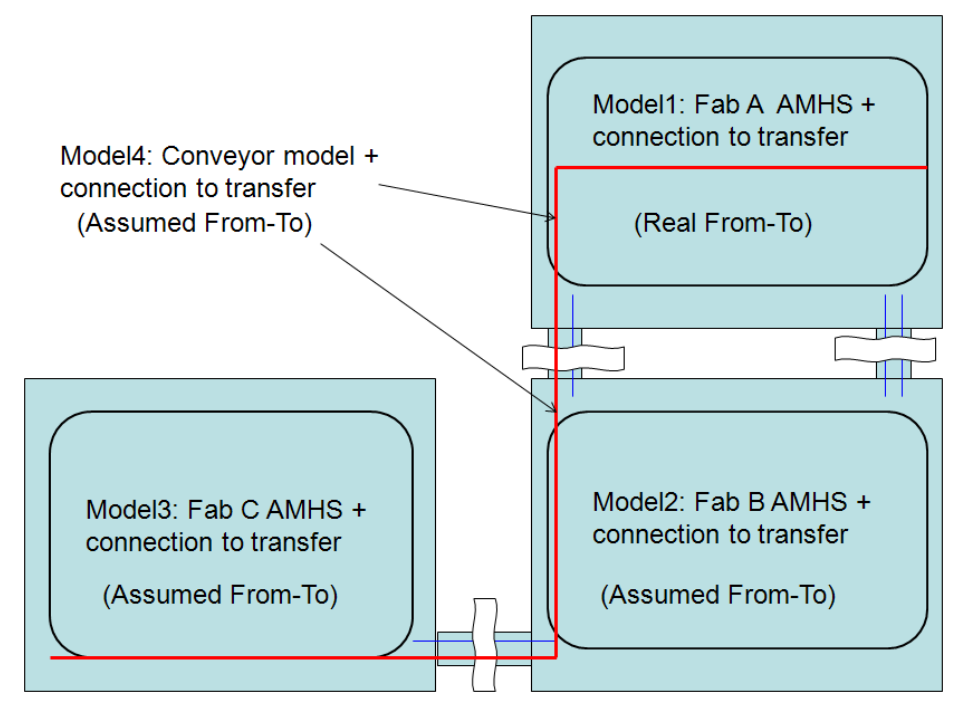

Figure 2: Segregated Dynamic Models Concept

Another way to keep the models segregated and also mitigate risk about the assumptions from the other fabs is to interconnect them using communication software (e.g. Model Communication Module) or using input/output files (e.g. drop off arrival rate at transfer station from other model) back and forth between different models. This approach could be especially useful when wafer carriers have to change from one system to another (e.g. from vehicle system to conveyor system), although this will also result in slower run time and communication time between models (e.g. when using Model Communication Module). The usefulness of this kind of interconnection is questioned though when the scope includes connecting the same types of systems (e.g. same version of a vehicle system) in different buildings, as it would miss the important aspects of vehicle balancing better captured with fully interconnected model approaches.

\subsection{Integrated Dynamic Models}

This kind of modelling is the most ambitious, but also the most complex and time consuming; both from the model building and model running perspective. However, it does represent the least risk in regards to results and input assumptions. In this approach, the AMHS from different buildings/fabs as well as all the interconnections (vehicles and/or conveyors) are simulated as a single model and areas of hand-shake between different systems are modelled (e.g. between conveyor and vehicle systems). One example is the case of two different fabs interconnected using a "same vehicle" based AMHS. In a segregated modelling 
approach, a vehicle would travel from origination point in one fab to a "transfer point" between the two fabs where the wafer carrier would be dropped off and the vehicle would be free to search for work in the original fab again. In the real AMHS however, the vehicle would travel to destination fab, drop off the wafer carrier, and then most likely would look for work or park at the destination fab. This situation and the corresponding necessary vehicle balancing logic can only be represented if the two fabs' AMHS systems are simulated in a single model. Failing to do so could lead to overlooking potential problems with the vehicle balancing logic or vehicle utilization. For situations where the change from one fab to another fab also includes a change of AMHS, the need of full interconnection might be less obvious but still would help reduce the risks associated with the arrival rate assumptions coming from the other fab. Better transporting times (for average values as well as peak $/ 95^{\text {th }}$ Percentile) and more accurate vehicle utilization statistics will be available from this approach when compared with the previous two approaches.

Figure 3 shows an example of an integrated (or quasi-integrated) dynamic approach for a multiple fab interlink design. The models were used for evaluating three different AMHS designs required to support three possible expansions in a given campus. The network based analysis (section 4.1) could be used to generate these designs out of a broader range of potential layouts. Design 1 and 2 consist of vehicle based AMHS connecting multiple buildings, and design 3 represents a vehicle based system plus a conveyor based AMHS interlink. For simplification purposes, the AMHS in the original fab and the interconnections were simulated in detail, but for the new fab the picks/drops to/from original fab were simulated, but intrafab moves were omitted. In spite of the simplifications, the integrated models had over 15,000 moves per hour and approximately 900 vehicles. Simulation run times were between $1.7 \mathrm{x}$ and $2.3 \mathrm{x}$ of actual times. This approach allowed for detailed study and statistics collection on the interconnecting links as well as the input/output areas in the original fab, but lacked detail in the study of the input/output areas of the new fab. Nevertheless, the approach was preferred to a completely segregated model approach because of the insight it provided about the interconnecting areas. It was also preferred over a completely integrated approach (with new fab simulated in detail) because of the faster model build and run times. AutoMod 12.4 simulation software based on 64 bit processing was used for these models.

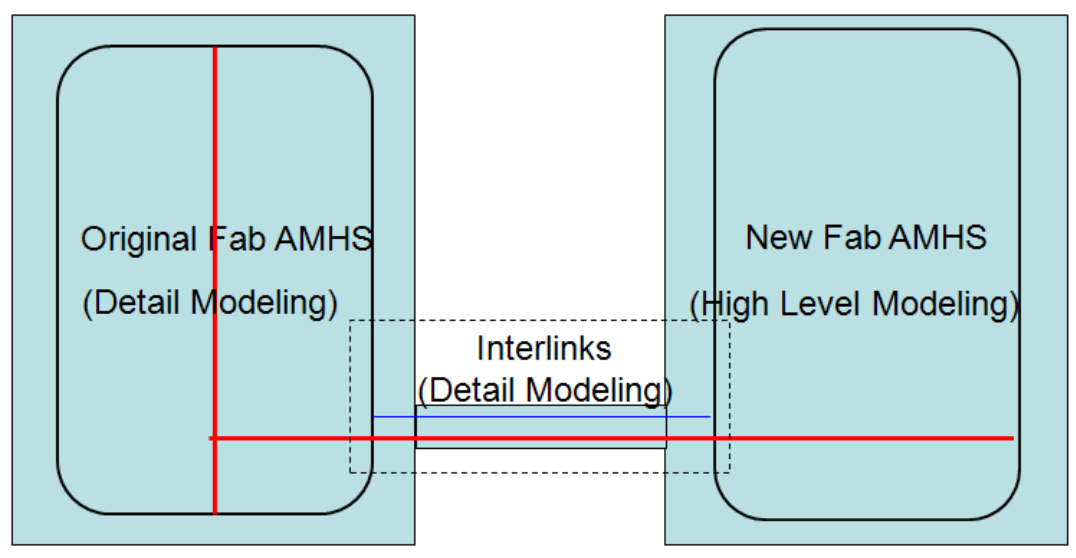

Figure 3: Integrated Dynamic Model Concept

\subsection{Pros/Cons of Each Approach}

Table 3 shows the pros/cons of the three approaches discussed in this paper as well as the use cases recommended. The network approach is fast to build and to run and allows for interconnecting several fabs together, but results will be limited by how well the designer understands the specific limitations of an AMHS (e.g. after having extensive experience with the dynamic modelling and real operations of that AMHS). It was agreed that this approach was suitable during high level design phase and when good understanding of the limitations of the AMHS exists. 
Segregated and integrated dynamic approaches are more suitable for detail design models than network approach as they allow for detail investigation of logic and behaviour of the AMHS. The segregated dynamic approach models are faster to build and run and are easier to maintain and manage, but rely heavily on assumptions about inputs from other fabs and in some cases might miss important logic (as the vehicle balancing logic between buildings). Taking into account the high cost of AMHS (typically several million dollars) and the cost of potential future disruptions in manufacturing, designers should consider ways to mitigate this risk. Risk mitigation could be accomplished by using a more complex fully integrated dynamic modelling approaches. In some cases, the benefits of using an integrated dynamic approach are more obvious. For example, when a single AMHS will be used to support different fabs vs. other cases when a different AMHS will be used to support different fabs.

Table 3: Pros/Cons of Network Approach, Segregated Dynamic Modeling and Integrated Dynamic Modeling

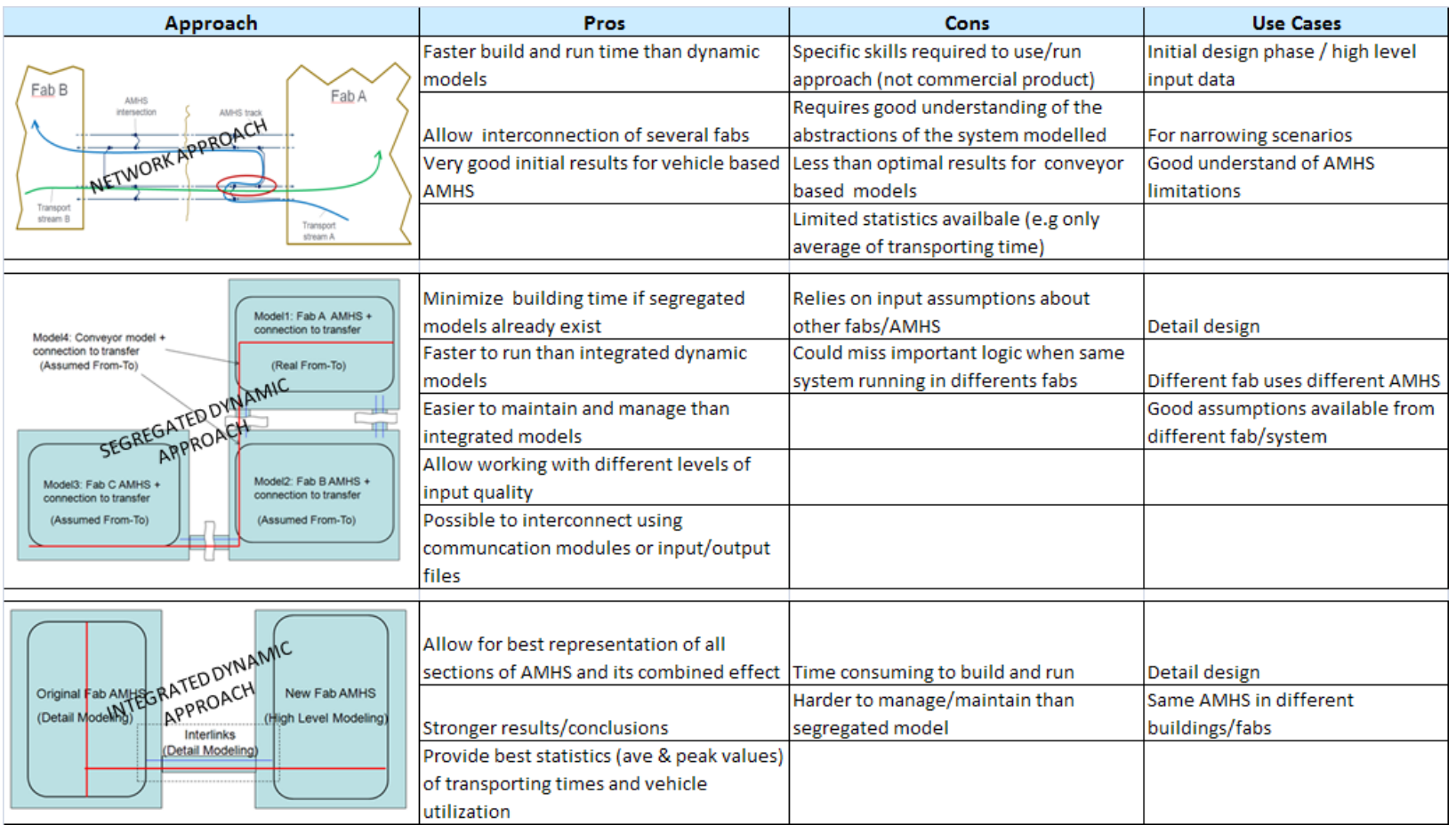

\section{SUMMARY AND CONCLUSIONS}

This paper presented three different options to evaluate AMHS interconnection designs for multiple fabs and discussed a simplistic methodology to forecast interfab moves. For high level design, the application of a static flow based approach called the Network approach produced very similar results to those obtained by more complex dynamic models, but only for the case of a well known vehicle based AMHS. For detailed design, more complex models based on segregated or integrated approaches are recommended. It was found that segregated dynamic approaches are more suitable for cases with a change of AMHS system between buildings, especially if a communication strategy between models is being considered. Integrated dynamic models are considered more suitable for cases where a single AMHS system is being used in multiple buildings. Today's 64 bit software will facilitate implementation and will reduce risks related to the AMHS logic implementation in different buildings.

Careful consideration of the AMHS design methodologies addressed in this paper is recommended to ensure the best return on investment for site capacity expansion planning. The authors believe further re- 
search is needed in the creation and validation of interfab move prediction models and in the evaluation, optimization, and impact of interconnected AMHS designs on combined fab performance.

\section{REFERENCES}

Floyd, R. W. 1962. Algorithm 97 (SHORTEST PATH). In Communications of the ACM 5, 6: 345.

Gaxiola, G., Hennessy, L., Glüer, D., Stachura, P., Christensen, E. 2004. Evaluation of an Overhead System as an Alternate Loop to an Interconnected Hoist System. 2004 Brooks Worldwide Automation Symposium, (no page numbers).

Hammel, C., A. Flemming, F. Schulze, K. Peters. 2008. Application of Methods from Complex Network Theory to MHS Layout Optimization. In Progress in Material Handling Research: 2008, 526-543.

Jimenez, J., Kim, B., Fowler, J., Mackulak, G., Choung, Y and Kim, D. 2002. Operational Modeling and Simulation of an Inter-Bay AMHS in Semiconductor Wafer Fabrication. In Proceedings of the 2002 Winter Simulation Conference, 1377-1382.

Jimenez, J., Mackulak, G. and Fowler, J. 2005. Efficient Simulations for Capacity Analysis and Automated Material Handling System Design in Semiconductor Wafer Fabs. In Proceedings of the 2005 Winter Simulation Conference, 2157-2161.

Jimenez, J., Mackulak, G. and Fowler, J. 2008. Levels of Capacity and Material Handling System Modeling for Factory Integration Decision Making in Semiconductor Wafer Fabs. IEEE Transactions on Semiconductor Manufacturing, Vol. 21, No. 4, November 2008, 600-613.

Jimenez, J., Bell, M., Adikaram, Ch., Davila,V., Wright, R and Grosser, A. 2010. AMHS Factors Enabling Small Wafer Lot Manufacturing in Semiconductor Wafer Fabs. In Proceedings of the 2010 Winter Simulation Conference, 2575-2585.

Kiba, J., Lamiable, G., Dauz`ere-P'er`es, S., and Yugma, C. 2009. Simulation of a Full 300mm Semiconductor Manufacturing Plant with Material Handling Constraints. In Proceedings of the 2009 Winter Simulation Conference, 1601-1609.

Kohn, R., Noack, D., Mosinski, M., Zhou,Z. and Rose, O. 2009. Evaluation of Modeling, Simulation and Optimization Approaches for Work Flow Management in Semiconductor Manufacturing. In Proceedings of the 2009 Winter Simulation Conference, 1592-1600.

Kondo, H. 2008. Impacts of Small Lot Manufacturing on AMHS. 2008 International Symposium on Semiconductor Manufacturing (2008 ISSM). 93-96.

Miller, L., Bradley, A., Tish, A., Jin, T., Jimenez, J. and Wright, R. 211. Simulating Conveyor-Based AMHS Layout Configurations in Small Wafer Lot Manufacturing Environments. In Proceedings of the 2011 Winter Simulation Conference, 1944-1952.

Nazzal, D. and McGinnis, L. 2006. An Analytical Model of Vehicle-Based Automated Material Handling Systems in Semiconductor Fabs. In Proceedings of the 2006 Winter Simulation Conference, 18711879.

Nazzal, D., Carlo H., Johnson, A. and Jimenez, J. 2008. An Analytical Model for Conveyor Based AMHS in Semiconductor Wafer Fabs. In Proceedings of the 2008 Winter Simulation Conference, 2148-2155.

Schöps, M., C. Hammel. 2012. Netzwerktheoretische Optimierung des Materialflusses in der Halbleiterproduktion. In Information als Veränderungstreiber - Technische \& Organisatorische Aspekte: Tagungsband: Institutskolloquium 2012 des Instituts für Technische Logistik und Arbeitssysteme, Edited by M. Schmauder, T. Schmidt, S. Rank, S. 74-81. - ISBN 978-3-86780-257-4.

Sturm, R., Seidelmann, J., Dorner, J. and Reddig, K. 2003. An Approach to Robust Layout Planning of AMHS. In Proceedings of the 2003 Winter Simulation Conference, 1366-1372.

Warshall, S. 1962. A Theorem on Boolean Matrices. In Journal of the ACM 9, 1: 11-12.

Zimmerhackl, O., Rothe, J., Schmidt, K., Marshall, L. and Honold, A. 2007. The Effects of Small Lot Manufacturing on AMHS Operation and Equipment Front-End Design. International Symposium on Semiconductor Manufacturing (2007 ISSM), 1-5. 


\section{AUTHOR BIOGRAPHIES}

GABRIEL GAXIOLA is a member of GLOBALFOUNDRIES' Manufacturing Technology organization where he leads the modeling and simulation activities. He has a combined experience of 14 years in consulting and semiconductor industry. In the past he has been part of Qimonda, CH2M-Hill and Brooks (PRI) Automation teams. He has a MS degree in Industrial Engineering from Arizona State University and his e-mail address is gabriel.gaxiola@GLOBALFOUNDRIES.com.

ERIC CHRISTENSEN is a member of GLOBALFOUNDRIES' Manufacturing Technology organization where he leads the integration and development of Automation Technology solutions for the company. He has a combined 26 years of semiconductor manufacturing systems experience at AMD and GLOBALFOUNDRIES including a 2 year assignment at SEMATECH. Eric has a BS degree in Mechanical Engineering from the University of Texas and can be contacted via email @ eric.christensen@GLOBALFOUNDRIES.com.

CHRISTIAN HAMMEL is a PhD student at Technische Universität Dresden. He is member of the scientific staff at the Professorship of Logistics Engineering. Having received his Diploma (similar to M.S. degree) in Applied Mathematics (Technomathematik) in 2007 from Technische Universität Dresden his research interests include the application of analytical methods to optimize existing AMHS as well as to support the design phase. His email address is christian.hammel@tu-dresden.de.

PAUL STACHURA of Stachura Enterprises, LLC has over 33 years of experience in the semiconductor industry. He has a BS degree in Physics from the University of Illinois. His work began in semiconductor manufacturing and progressed to his current job as an industry consultant for semiconductor factory design and manufacturing equipment layout. He has worked in the US, Europe, Asia, Japan, and the Middle East. He is based in Austin, TX, USA. His email address is pgstachura@gmail.com. 